\title{
Efectividad según duración de la psicoterapia en un centro de atención psicológica para universitarios
}

\section{Effectiveness of psychotherapy according to the duration of treatment in an university counseling center}

\author{
Beatriz Painepán ${ }^{1} \quad$ Walter Kühne \\ Departamento de Promoción de la Salud Psicológica \\ Universidad de Santiago de Chile \\ (Rec: mayo de 2012 - Acep: junio de 2012)
}

\begin{abstract}
Resumen
Se presentan los resultados de una investigación que aborda la efectividad de la psicoterapia, de acuerdo a la duración del tratamiento en una muestra de estudiantes que acuden a un centro de atención psicológica universitario. El tipo de estudio es de medidas repetidas o longitudinal y el diseño es cuasi experimental. La muestra es intencionada y estuvo compuesta por 21 personas de ambos sexos. Se ha utilizado el cuestionario Outcome Questionnaire de Lambert (OQ-45.2). Se concluye que el tratamiento psicoterapéutico es efectivo. Los consultantes, en promedio, cambian de presentar puntuaciones clínicas a puntuaciones no clínicas a partir de la octava sesión.
\end{abstract}

Palabras clave: psicoterapia, efectividad y duración de la terapia.

\begin{abstract}
This paper presents the results of a research about effectiveness of psychotherapy according to the duration of treatment in a sample of students attending an university counseling center. The type of study is repeated measures or longitudinal and quasi-experimental design. The sample is intentional and consisted of 21 subjects of both sexes. It was used Outcome Lambert Questionnaire (OQ-45.2). The conclusion is that a psychotherapy is effective. The consultants changed from clinical scores to non-clinical from the 8th session.
\end{abstract}

Keywords: psychotherapy, effectiveness and psychotherapy length.

\footnotetext{
Correspondencia dirigida a: Beatriz Painepán Sandoval. Departamento de Promoción de la Salud Psicológica. Universidad de Santiago de Chile. E-mail: beatriz.painepan@usach.cl.
} 


\section{Introducción}

Desde el clásico artículo de Eysenck (1952), la efectividad de la psicoterapia ha sido tema de interés de clínicos e investigadores. Las afirmaciones de Eysenck, como que un paciente tenía la misma probabilidad de mejorar si estaba en la lista de espera o en psicoterapia, han sido refutadas por la investigación subsiguiente que ha arrojado contundente evidencia. Hasta llegar al punto en que Luborsky hace una comparación con el veredicto del pájaro Dodó: todas las psicoterapias son efectivas y deben ganar premio (1975). Sin embargo, tal como en el libro de Lewis Carroll, a Alicia le parece poco lógico que todos ganen premios, los investigadores también pusieron en duda la apuesta de Luborsky y continuaron poniendo a prueba las distintas psicoterapias, llegando a resultados más complejos. Actualmente la psicoterapia basada en la evidencia se ha enfocado en señalar qué tipo de terapia es más efectiva para diagnósticos o pacientes en particular (Chambless y Hollon, 1998; Chambless y Ollendick, 2001).

Es en este contexto de investigación donde surgen distintas preguntas sobre las condiciones en las cuales funciona la psicoterapia. El presente estudio se centra en uno de tales aspectos, su duración.

La pregunta por la efectividad debe combinarse con la evaluación de la eficiencia del procedimiento. Dos psicoterapias igualmente efectivas pueden tener costos muy distintos en función, por ejemplo, de la cantidad de sesiones requerida para lograr el cambio. Si bien hay evidencia que señala que la terapia de tiempo limitado y sin límite tiene una efectividad equivalente (Orlinsky, Ronnestad y Willutzki, 2003), se requiere saber cuál es el tiempo limitado óptimo.

El problema de la eficiencia de la psicoterapia surge principalmente a partir de la necesidad de distribuir los recursos limitados de manera racional, con el fin de beneficiar a la mayor población posible (Máiquez, BlancoVillaseñor, Rodrigo y Vermaes, 2000). Los sistemas de salud y bienestar de la población generalmente no cuentan con los recursos necesarios para dar cobertura a todas las personas que lo requieren. Esto plantea el problema de determinar cuántas sesiones son óptimas para definir la duración de una psicoterapia de tiempo limitado.

Otro grupo de variables que se ha considerado para determinar bajo qué condiciones es efectiva la psicoterapia son las variables que corresponden a los consultantes. En esta línea, las características de poblaciones específicas aparecen como relevantes. Los estudiantes universitarios son una población sobre la que se realizan estudios específicos de prevalencia en trastornos mentales (Cova y cols, 2007 y Micin y Bagladi, 2011), especialmente considerando que los problemas de salud mental en esta población se han incrementado en la última década (Storrie, Ahern y Tuckett, 2010) al punto de presentar en algunos casos prevalencias mayores a la población general (Stallman y Shochet, 2009). Como consecuencia de esta realidad, algunas universidades brindan servicios de psicoterapia a sus estudiantes (López et al., 2010). No obstante, la gratuidad y las limitaciones de recursos hacen necesaria la optimización de éstos.

El presente estudio se centra en el funcionamiento de un centro de atención psicológica gratuito para estudiantes de la Universidad de Santiago de Chile, en el cual los recursos existentes no permiten disponer de horas suficientes para todos los consultantes. Esta problemática motiva la realización de un estudio de eficiencia para determinar cuál es la mejor cantidad de sesiones para fijar el límite de duración de los procesos de terapia. La relevancia de este estudio radica en que permitiría mejorar la distribución de los recursos disponibles para dar la mayor cobertura a la población solicitante.

El objetivo de esta investigación es determinar la cantidad de sesiones de psicoterapia óptima para fijar la duración de los procesos psicoterapéuticos. Es decir, cuál es la menor cantidad de sesiones en la cual la psicoterapia es efectiva. El estudio es de tipo explicativo y el diseño es cuasi experimental y longitudinal o de medidas repetidas sobre la misma muestra. La medición se realizó en cuatro momentos distintos durante la psicoterapia de los participantes.

La variable independiente es la efectividad de la terapia medida a través del Outcome Questionnaire (OQ) de Lambert, instrumento adaptado y validado a Chile por Von Bergen y De la Parra (2002). Por otro lado, la variable dependiente es la cantidad de sesiones de psicoterapia. Se realizaron mediciones de resultado con el OQ en las sesiones 1, 4, 8 y 12 .

En la presente investigación se espera encontrar que la cantidad de sesiones óptima es dentro de las primeras ocho, dado que en un estudio anterior en el mismo centro se estableció que el $84.22 \%$ de las terapias terminan dentro de las primeras diez sesiones y el promedio de duración es 7,8 sesiones (López et al., 2010).

\section{Método}

\section{Muestra}

La muestra es intencionada de un grupo de pacientes del centro de salud psicológica. Participaron 
21 estudiantes universitarios de ambos sexos. Estas personas fueron elegidas entre quienes se atendían en el centro de atención y que cumplieran con el criterio de tener doce sesiones de psicoterapia realizadas, excluyendo a quienes presentaran patologías mentales severas tales como esquizofrenia o algún otro tipo de psicosis.

\section{Instrumento}

Se utilizó el Cuestionario para la Evaluación de Resultados y Evolución en Psicoterapia u Outcome Questionnaire de Lambert (OQ-45.2), adaptado y validado en Chile por Von Bergen y De la Parra (2002). Desarrollado por Lambert y Berlingame en 1996 en Estados Unidos. El cuestionario consta de 45 ítems de autorreporte en escala Likert y evalúa tres áreas: Sintomatología o malestar subjetivo (25 ítems), Relaciones interpersonales (11 ítems) y Rol social ( 9 ítems). El tiempo promedio del cuestionario autoaplicado es de 15 minutos. En la adaptación de Von Bergen y De la Parra la consistencia interna para el puntaje total del OQ medido con alfa de Cronbach es de 0.93. En la validación chilena del instrumento se establece que el Puntaje de Corte (PC) que diferencia un estado clínico de un estado saludable es 73 puntos. Puntajes iguales o superiores a 73 puntos significa que los sujetos presentan resultados disfuncionales que indican su necesidad de atención psicoterapéutica. Los puntajes de corte por subescalas son 43 puntos en Sintomatología, 16 puntos en Relaciones interpersonales y 14 puntos en Rol social.

\section{Procedimiento}

Los participantes fueron seleccionados al azar de entre los pacientes atendidos en el servicio de salud psicológica de la universidad. Ellos accedieron a participar voluntariamente de la investigación firmando un consentimiento informado sobre el uso de los resultados de sus cuestionarios. El instrumento fue aplicado en la primera, cuarta, octava y doceava sesión. Los resultados fueron digitados en un computador Compaq Presario CQ5704. Y la información obtenida fue analizada mediante el programa estadístico SPSS para ciencias sociales, en versión 17.0 y se utilizó el mismo computador antes señalado.

\section{Análisis de datos}

Se identificaron los estadígrafos descriptivos para los resultados de cada medición. Los coeficientes utilizados fueron los de Shapiro-Wilks para evaluar normalidad, la Prueba de Rachas para independencia, la aditividad fue evaluada con el coeficiente de Freedman, el coeficiente de Bartlett para evaluar esfericidad y el contraste de hipótesis con el coeficiente de $\mathrm{T}$ de Hottelling. Para los contrastes post-hoc se utilizó el ajuste de Bonferroni y se efectuó la Prueba de Contraste Polinómico graficando los resultados significativos. El nivel de significancia propuesto fue de $\mathrm{p}$ menor 0,05 .

\section{Resultados}

Se presentan los resultados obtenidos a un nivel descriptivo para cada una de las cuatro mediciones realizadas a los 21 sujetos participantes en la muestra. Como se observa en la tabla 1, la media de la muestra en la primera sesión se encuentra sobre el puntaje de corte ( $\mathrm{PC}=73$ puntos) y en la sesión 12 el puntaje de la media del grupo está bajo el PC, es decir, no clínico.

\section{Análisis de los supuestos}

Previo al contraste de la hipótesis de investigación se revisan los supuestos de normalidad, independencia, aditividad y esfericidad en los resultados obtenidos. Conforme a los valores de los estadígrafos observados

Tabla 1.

Estadígrafos descriptivos y estimadores de confianza para la variable sintomatología clínica general en pacientes que asisten a terapia psicológica.

\begin{tabular}{lcccc}
\hline Estadígrafos & Media & Error Típico & \multicolumn{2}{c}{ Intervalo de Confianza } \\
& & & Límite inferior & Límite superior \\
\hline Medida sesión 1 & 76,524 & 5,852 & 64,316 & 88,732 \\
Medida sesión 4 & 72,238 & 4,985 & 61,840 & 82,636 \\
Medida sesión 8 & 66,524 & 4,184 & 57,797 & 75,251 \\
Medida sesión 12 & 61,333 & 4,415 & 52,124 & 70,543 \\
\hline
\end{tabular}

* Nivel de confianza: 95\%. 
para el supuesto de la normalidad multivariada, se asume que al mantenerse la hipótesis de normalidad de las puntuaciones univariadas en la variable efectividad de la psicoterapia en los cuatro momentos distintos de medición, existen condiciones necesarias (aunque no suficientes) para asumir normalidad conjunta multivariada.

En relación al supuesto de aleatoriedad de las puntuaciones obtenidas, se asume que existe independencia univariada en las puntuaciones para la variable dependiente en estudio, efectividad, en los cuatro momentos del tiempo (sesiones 1, 4, 8 y 12). Lo que se indica anteriormente confirmaría la existencia de aditividad, dado que se mantiene la hipótesis nula que señala que no hay interacción entre los niveles del tratamiento aplicado y las puntuaciones de la variable efectividad a través del tiempo.

Tabla 2.

Evaluación de supuestos paramétricos para la variable Efectividad del Tratamiento Psicoterapéutico.

\begin{tabular}{lllll}
\hline Supuesto & \multicolumn{1}{c}{$\mathrm{T} 1$} & \multicolumn{1}{c}{$\mathrm{T} 2$} & \multicolumn{1}{c}{$\mathrm{T} 3$} & $\mathrm{~T}$ \\
\hline Normalidad & $\mathrm{SW}(21)=0.96$ & $\mathrm{SW}(21)=0.933$ & $\mathrm{SW}(21)=0.923$ & $\mathrm{SW}(21)=0.936$ \\
& $(\mathrm{p}=0.681)$ & $(\mathrm{p}=0.158)$ & $(\mathrm{p}=0.098)$ & $\mathrm{z}=0.179)$ \\
Independencia & $\mathrm{Z}=-.360$ & $\mathrm{Z}=-1.145$ & $\mathrm{Z}=-.817$ & $\mathrm{Z}=-.193$ \\
(Rachas) & $(\mathrm{p}=0.719)$ & $(\mathrm{p}=0.252)$ & $(\mathrm{p}=0.414)$ & $(\mathrm{p}=0.847)$ \\
Aditividad & $\mathrm{F}(1)=2.800$ & $\mathrm{~F}(1)=2.800$ & $\mathrm{~F}(1)=2.800$ & $\mathrm{~F}(1)=2.800$ \\
& $(\mathrm{p}=0.100)$ & $(\mathrm{p}=0.100)$ & $(\mathrm{p}=0.100)$ & $(\mathrm{p}=0.100)$ \\
Esfericidad & $\chi^{2}(9)=42.590$ & $\chi^{2}(9)=42.590$ & $\chi^{2}(9)=42.590$ & $\chi^{2}(9)=42.590$ \\
Bartlett & $(\mathrm{p}=0.000)$ & $(\mathrm{p}=0.000)$ & $(\mathrm{p}=0.000)$ & $(\mathrm{p}=0.000)$
\end{tabular}

Finalmente, en relación al supuesto de esfericidad de la matriz de varianzas covarianzas, se observa que la hipótesis nula de homogeneidad de varianzas a través del tiempo no se mantiene. Por lo tanto, el contraste que se utiliza para analizar la hipótesis del diseño de investigación es la prueba multivariada T de Hottelling.
El contraste de hipótesis se realizó utilizando la prueba $\mathrm{T}$ cuadrado de Hottelling obteniéndose un $\mathrm{F}=3.199$ con una significancia $=0.048$. Con esto se señala que al menos uno de los pares de las medidas comparadas presentan diferencias estadísticamente significativas entre ellos. El tamaño del efecto (eta

Tabla 3.

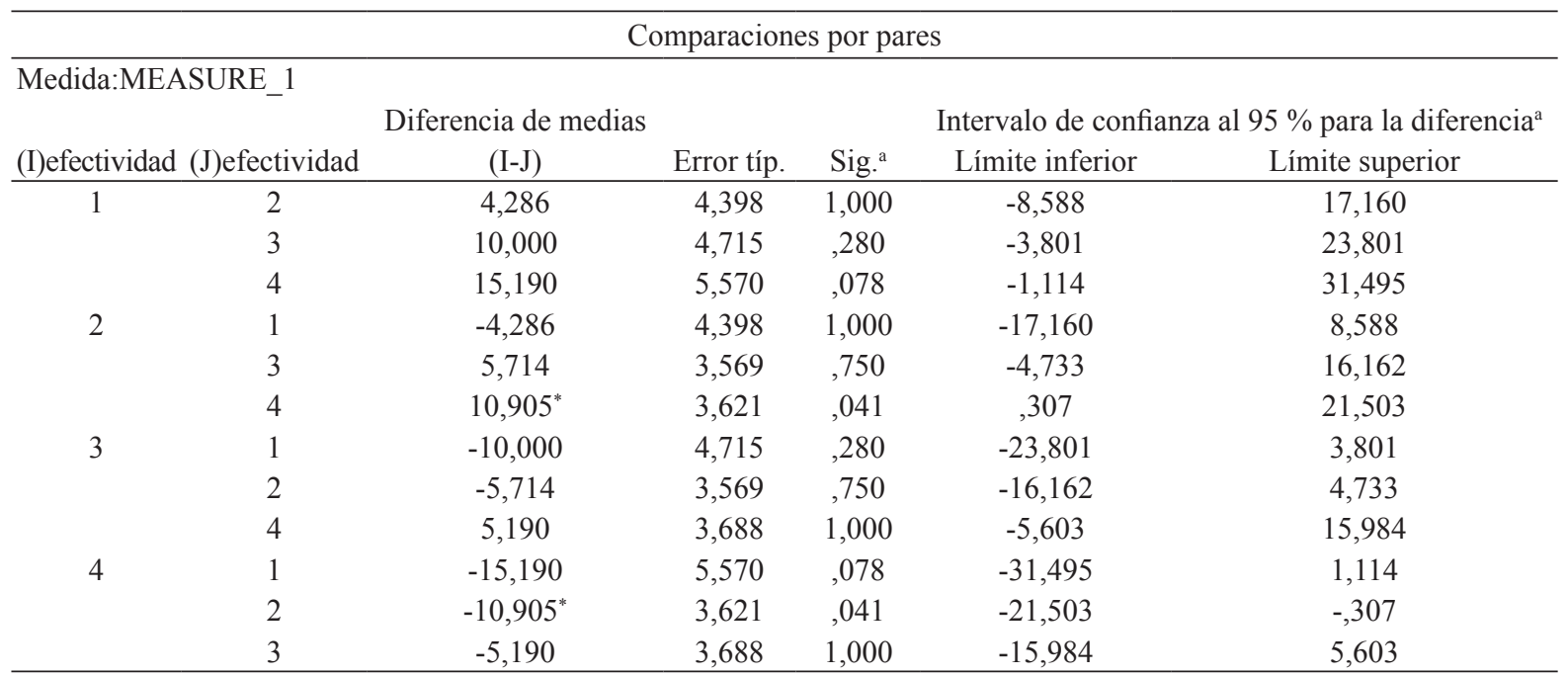

Basadas en las medias marginales estimadas.

a. Ajuste para comparaciones múltiples: Bonferroni.

*. La diferencia de medias es significativa al nivel ,05. 
cuadrado parcial) encontrado es de .348 y la potencia observada es de .637

Para identificar los grupos que al compararse presentan diferencias significativas se utilizan los Contrastes Post hoc, realizando el ajuste con el coeficiente de Bonferroni.

Las diferencias estadísticamente significativas se encuentran al comparar las medidas de la medición 2 ( $4^{\mathrm{a}}$ sesión) con la medición 4 ( $12^{\mathrm{a}}$ sesión), con una diferencia de medias de 10,905 puntos $(\mathrm{p}=0.041)$ y un $\alpha=0.05$. El intervalo de confianza identificado incluye los valores $(.307$ - .21,503). Como los valores encontrados son ambos positivos se confirma la negación de la hipótesis de nulidad entre los valores comparados.

Posteriormente, se realizan contrastes polinómicos para identificar el comportamiento de la variable dependiente estudiada, obteniéndose una función lineal con un valor $\mathrm{F}=8,792$ ( $\mathrm{p}=0,008)$. El tamaño del efecto o eta al cuadrado corresponde a 0.305 y la potencia observada es 0.805 .

En el gráfico de contraste lineal se observa un comportamiento de los puntajes de la variable efectividad de tipo lineal inversa, es decir, a través del tiempo se observa mayor efectividad del tratamiento ya que los puntajes obtenidos mediante el instrumento OQ disminuyen progresivamente.

\section{Resultados por subescalas}

Los análisis de supuestos de las puntuaciones de las subescalas obtienen los mismos resultados que para el análisis del puntaje total.

El análisis por subescalas reporta sólo una diferencia significativa entre la segunda medición y la cuarta medición $(\mathrm{F}=5.028(3), \mathrm{p}=0.011, \alpha=0.05)$ en la subescala Sintomatología. De este modo, el comportamiento de los puntajes de esta subescala es similar al del puntaje total. El comportamiento de esta variable también adquiere una forma de función lineal. Las otras dos subescalas, Relaciones Interpersonales y Rol Social, no arrojan diferencias estadísticamente significativas entre las distintas mediciones.

En cuanto al Puntaje de Corte (PC), tal como se observa en la tabla 4, en la subescala Sintomatología la media de la muestra en la primera medición se encuentra sobre el puntaje de corte. En las siguientes mediciones disminuye progresivamente. En las sesiones 8 y 12 el puntaje promedio se ubica bajo el puntaje de corte.

Los puntajes promedio de subescalas Relaciones Interpersonales (RI) y Rol Social (RS) disminuyen pero
Gráfico 1.

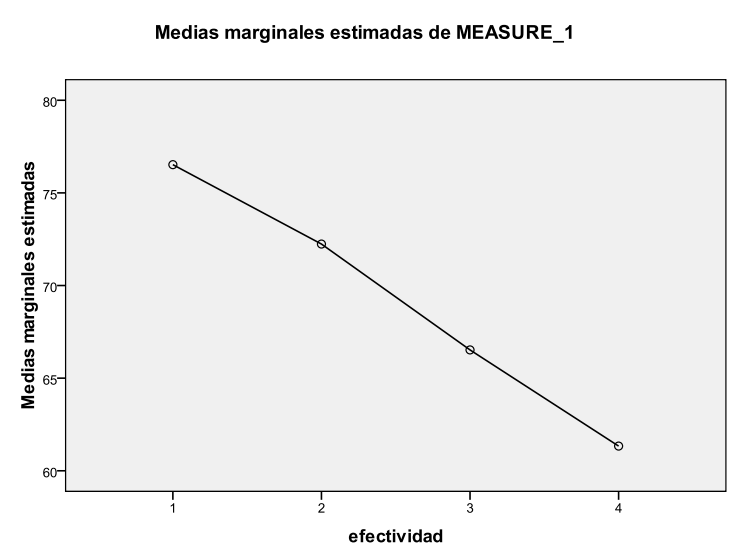

Tabla 4.

Puntaje de corte y puntajes promedios por subescala.

\begin{tabular}{lccccc}
\hline & $\begin{array}{c}\text { Puntaje de } \\
\text { Corte }\end{array}$ & $\begin{array}{c}\text { Sesión } \\
1\end{array}$ & $\begin{array}{c}\text { Sesión } \\
4\end{array}$ & $\begin{array}{c}\text { Sesión } \\
8\end{array}$ & $\begin{array}{c}\text { Sesión } \\
12\end{array}$ \\
\hline Sint & 43 & 45,38 & 44.10 & 38.86 & 33.9 \\
RI & 16 & 17.61 & 16.76 & 15.71 & 16.23 \\
RS & 14 & 13.52 & 11.38 & 11.95 & 11.19 \\
\hline
\end{tabular}

no de una manera estadísticamente significativa. Si bien en términos generales las puntuaciones de estas subescalas tienden a disminuir, se observa que oscilan en un margen muy estrecho de puntuaciones, variando en no más de dos puntos. Si bien RI tiende a disminuir en sus puntuaciones, éstas tienden a mantenerse en torno al PC de 16 puntos. Por su parte, las puntuaciones en RS oscilan y tienden a disminuir manteniéndose siempre por debajo del PC.

\section{Discusión}

A partir de los análisis estadísticos realizados se observan diferencias estadísticamente significativas en los resultados obtenidos al comparar la cuarta y la doceava sesión.

Este comportamiento irregular de la variable en estudio, efectividad en relación a lo esperado, puede encontrarse mediado por la eventual presencia de variables covariadas que pudieran estar incidiendo en los resultados. En este sentido sería recomendable realizar un nuevo estudio aumentando la muestra o la cantidad de participantes para confirmar o no una réplica de los resultados obtenidos. También es necesario considerar, por ejemplo, las posibles influencias implicadas en que las intervenciones se hayan efectuado por distintos 
psicoterapeutas, variable que no está controlada en el análisis de los resultados.

Sin embargo, a partir de las consideraciones técnicas y de acuerdo al instrumento aplicado, es posible afirmar que la psicoterapia es efectiva en disminuir los indicadores clínicos de los consultantes en el centro de atención para universitarios. Si bien se observa una consistente mejora en los resultados en el transcurso de las sesiones, la mayor efectividad está significativamente establecida para las 12 sesiones.

A las 8 sesiones los consultantes en promedio ya están bajo la línea de corte clínico. Es decir, la mayoría ya no presenta indicadores clínicos.

Estos resultados permiten concluir que, en términos de eficiencia, la terapia limitada a 12 sesiones es preferible a la terapia sin límite de tiempo. En la muestra del estudio los pacientes han dejado de presentar indicadores clínicos.

En la sesión número 12 todos los sujetos están bajo el puntaje de corte, es decir, ninguno tiene puntajes clínicos y de acuerdo a la interpretación del OQ podrían ser dados de alta.

La secuencia que se utilizó para la investigación es cíclica. Dada la naturaleza de las intervenciones psicoterapéuticas no manualizadas no es posible utilizar una secuencia balanceada, es decir, programar las intervenciones de modo tal que permita alternar los distintos tipos de intervenciones en los distintos momentos de medición.

Otra limitación del estudio es que la cantidad de sujetos obliga al uso de técnicas estadísticas no paramétricas.

Los aportes e implicancias del estudio se relacionan con la posibilidad de presentar los resultados obtenidos en las psicoterapias del centro de atención estudiantil, la posibilidad de comparar estos resultados a otros centros similares y aportar en un área de investigación donde actualmente existe escasa información teórica.

\section{Referencias}

Chambless, D. y Ollendick, T. (2001). Empirically supported psychological interventions, Annual Review of Psychology, 52, 685-716.

Chambless, D. y Hollon, S. (1998). Defining Empirically Supported Therapies, Journal of Consulting and Clinical Psychology, 66, 7-18.

Cova, F., Labial, W., Aro, M., Bonifetti, A., Hernández, M. y Rodríguez, C. (2007). Problemas de Salud mental en estudiantes de la Universidad de Concepción, Terapia Psicológica, 25, 105-112.

Eysenck, H. .J. (1952). The effects of psychotherapy: An evaluations. Journal of Consulting Psychology, 9, 140-146.

López, M., Kuhne, W., Pérez, P., Gallero, P. y Matus, O. (2010). Características de Consultantes y Proceso Terapéutico de Universitarios en un Servicio de Psicoterapia, Revista Iberoamericana de Psicología, 3, 99-107.

Luborsky, L., Singer, B. \& Luborsky, E. (1975). Comparative studies of psychotherapies: Is it true that "everybody has won and all must have prizes?" Archives of General Psychiatry, 32, 995-1008.

Máiquez, M., Blanco-Villaseñor, A., Rodrigo, M. y Vermaes, I. (2000). La evaluación de la eficiencia en la intervención familiar: Psicothema, 12, 533-542.

Micin, S. y Bagladi, V. (2011). Salud Mental en Estudiantes Universitarios: Incidencia de Psicopatología y Antecedentes de Conducta Suicida en Población que Acude a un Servicio de Salud Estudiantil, Terapia Psicológica, 29, 53-64.

Orlinsky, D., Ronnestad, M. y Willutzki, U. (2011). SPR Collaborative Research Program on the Development of Psychotherapists en Norcross, J., VandenBos, G. y Freedheim, D., History of Psychotherapy, Washington, DC: American Psychological Association.

Stallman, H. M. y Shochet, I. (2009). Prevalence of mental health problems in Australian university health services, Australian Psychologist, 44, 122-127.

Storrie, K., Ahern, K. y Tuckett, A. (2010). Students with mental health problems, International Journal of Nursing Practice, $16,1-6$.

Von Bergen, A. y De la Parra, G. (2002). OQ-45.2, Cuestionario para Evaluación de Resultados y Evolución en Psicoterapia: Adaptación, Validación e Indicaciones para su Aplicación e Interpretación, Terapia Psicológica, 20, 161-176. 